\title{
EFFECTS OF BUSINESS INTELLIGENCE APPLICATION IN TOLLING SYSTEM
}

\author{
Gordana Radivojević ${ }^{1,2}$, Bratislav Lazić ${ }^{2}$, Gorana Šormaz ${ }^{3}$ \\ ${ }^{1}$ University of Belgrade, Faculty of Transport and Traffic Engineering, Vojvode Stepe 305, 11000, \\ Belgrade, Serbia \\ ${ }^{2,3}$ University of Belgrade, Mihajlo Pupin Institute, Volgina 15, 11000 Belgrade, Serbia
}

Received 7 November 2014; accepted 21 December 2014

\begin{abstract}
Tolling system is a complex information and communication system that provides toll charging on highway sections. Traffic Management and Supervision System - TMSS enables the communication integration of all the elements of the system, data collection and data processing and generation of the set of reports, based on which it is possible to monitor the operation of the system both in on-line as well as in off-line regime and to control the realization of all the functions in the system. TMSS is developed on the modern technological platform which includes the Business Intelligence technology - BI and the Data Warehousing technology - DWH. The main goal of this paper is to show the usage and effects of the Business Intelligence technology in the TMSS development and exploitation.
\end{abstract}

Keywords: business intelligence, data warehousing, tolling system, data processing, analytics and reporting.

\section{Introduction}

A tolling system is a complex system which, on the one hand enables toll charging on highway sections, and on the other, provides fast and high-quality usage of the highway. It is very important for the state - the owners of highways, to have a good and reliable system which enables high-quality functioning of toll charging in all sections. The drivers customers of highways, want the processes of toll collection to be fast and reliable, with the possibility of different payment methods. Thus, it is necessary to provide an integrated management and supervision system of toll collection.

The goal of this paper is to describe the effects of applying Business Intelligence $\mathrm{BI}$ in the development and exploitation of
Traffic Management and Supervision System - TMSS. The paper consists of five parts. The first part describes the tolling system. The second part describes TMSS and its basic modules. The third part outlines the application of Business Intelligence in the development of TMSS while the fourth part specifies the effects of the application. The conclusion forms the fifth part of this paper.

\section{Toll System Description}

The toll collection process is realized at toll plazas located at one or more highway sections. Each toll plaza has one or more lanes in which the following can be carried out: physical flow of vehicles (entrance and exit of vehicles to the highway), toll collection process, recording all data on vehicles, control of peripheral equipment

${ }^{1}$ Corresponding author: g.radivojevic@sf.bg.ac.rs 
and communication with toll plazas. The plaza is connected with all pertaining toll lanes and it enables the following: provides communication with lanes, collects data from the lanes and generates a collection of daily performance reports. Furthermore, the toll plaza is linked with the Central Level where all information on lanes is sent. The Central Level gathers, processes and stores data, enables supervision and monitoring of toll collection (Radivojević et al., 2013).

Toll collection system is a complex information system which combines hardware and software components. In view of organization, the toll collection system consists of three hierarchical levels: the toll lanes, the toll plaza and the central level (Mihajlo Pupin Institute, 2012). All three levels are in on-line or off-line communication (Fig. 1).

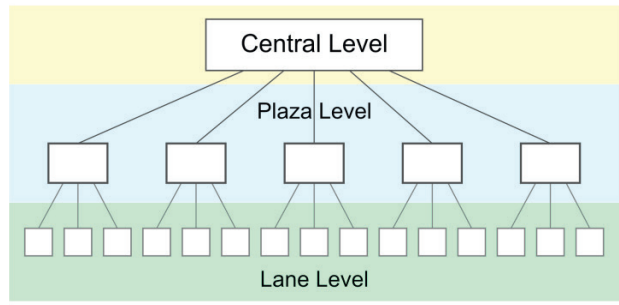

Fig. 1.

Hierarchical Layers of Toll System

The Central level receives, stores and processes all toll collection data. The main function of the Central Level is to connect the lower levels into an integral system, to communicate with external systems, manage and control the complete toll collection system. The Central level gathers data from the lower levels (toll plaza, toll lanes) and then all necessary system parameters are distributed to them. The
Central Level is also connected with external systems with which it exchanges various data sets. External systems include: business information system (ERP), banks (credit and debit card processing), sales points for mediums for toll payment (On-Board Unit OBU and Company Cards - CC), and others (Radivojević et al., 2013).

\section{The Toll Management and Supervision System}

TMSS enables supervision and management of toll collection in real-time. The basic goals when developing TMSS were:

- Developing a modern system which would provide on-line monitoring of toll collection process on all toll plazas and lanes on highways;

- Good work performances of the system in real-time;

- Work with large quantities of data and parallel execution of various functionalities without compromising performances, and

- Creating the possibility of expanding the system with new functionalities during exploitation.

An overview of the basic functionalities was made in the initial phase of designing the system and over time broadened in conformity with the process requirements and user's requests. The initial set of functionalities realized the following tasks:

- Connection of all elements on various hierarchical levels into an integral system;

- Connection with external systems (ERP, banks, sales points, etc.);

- Acquiring data from lower levels of system in real-time (on-line); 
- The possibility of importing data from lower levels in off-line mode of communication;

- Distribution of system parameter sets to lower levels of the system;

- Monitoring toll collection process in real-time;

- Monitoring state of equipment on all toll plazas and lanes;

- Generating work reports from all system segments;

- Distribution of reports to various groups of users; etc.

In order to realize such a set of tasks it was necessary to apply contemporary and modern technology which enables fast, highquality and reliable data base designing, communication protocols, web applications, reports and analytics, together with high performances of their exploitation.

TMSS is implemented on Oracle Fusion Middleware technology platform which consists of Oracle WebLogic application server and Oracle BI (Business Intelligence) platform for data warehousing (Data Warehousing - DWH) and analytical reports. Data layer is implemented as a mixed transaction (Online Transaction Processing - OLTP) and analytical (Online Analytical Processing - OLAP) model. Data are stored in Oracle $11 \mathrm{~g}$ data base (Oracle, 2013). Access to all system data and functions is protected by defining role of direct users.

TMSS is designed as a set of integrated modules which correspond to the organization structure of toll collection system. Each module encompasses a group of functionalities which refer to individual segments of work. The following present the basic TMSS modules (Fig. 2):

- Internal and External integration,

- System management,

- Toll charging,

- Financial and Customer Management, and

- Analytics and Reporting.

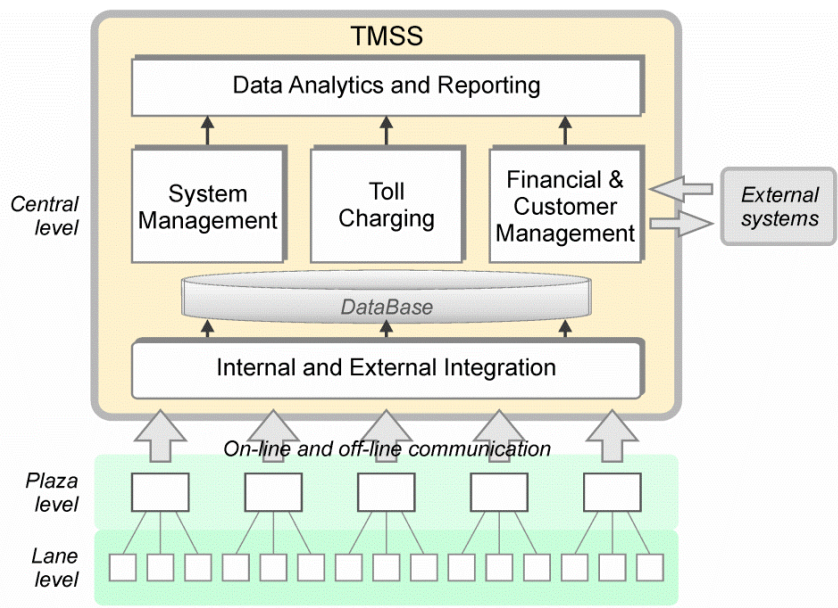

Fig. 2.

Architecture and Modules of the TMSS 
Internal and External integration enables information communication and data exchange among all levels within the toll collection system (Plaza, Toll and Central) as well as with external systems - ERP, banks, OBU and CC sales points, and other systems. Data exchange is carried out by way of messages by applying IMP TCP protocol and SOAP web service. Protocol adapters support on-line and off-line data transmission.

System management enables overall system supervision and physical configuration as well as monitoring state of equipment in the system. This module includes: system configuration management, system monitoring and supervision, maintenance management, security and users, and test management.

Toll charging module covers processing of all data relating to the physical process of toll collection and which are generated from the hierarchically lower levels. The basic components of the module are: managing business parameters, toll collection transaction processing, monitoring toll collectors and managing pre-magnetized cards.

Financial and Customer Management involves data processing on $\mathrm{OBU}$ and $\mathrm{CC}$ clients, their accounts and contractual relations. This module includes: management of finance and accounts, clients and sale, management of individual OBU and CC, forming gray and black lists of medium, and similar.

Analytics and Reporting includes data processing and generating report sets on the toll collection system. Analytics is based on DWH architecture and reporting is realized by applying Oracle BI technology.
DWH and BI enable generating reports for large quantities of data along with high performances of system work.

\section{Business Intelligence Application}

Business Intelligence - BI is a set of methods, processes, architectures and technologies, which transform raw data into meaningful and useful information and knowledge. BI is a contemporary technology of acquiring, storing and processing of large quantities of data. Data are acquired from various sources, stored and processed according to business system requirements, with high work performances in real-time. BI provides business information and various analyses of key business processes, making high-quality decisions on various managing levels and improving performances in the business system. BI applications include various forms of reports to enable high-quality and timely information to users on the key business processes in real and nearly real-time.

BI technology is used in developing TMSS for many reasons:

- Acquiring data from various sources (Toll lanes, Toll plazas, OBU and CC sales points, banks, ERP, and others),

- Data are acquired in real-time and/or imported in off-line regime,

- Processing of large quantities of data which can refer to any historical period,

- Monitoring various business processes on lower levels (Toll plazas and lanes),

- Reporting on all business processes on various levels of detail,

- Distribution of reports to users in appropriate formats according to concrete requirements,

- Data protection in system and good work performances of all functionalities in real-time, etc. 
DWH and a set of BI applications have been designed within the TMSS which realize the Analytics and Reporting module. DWH is a contemporary data base technology which presents the basis for development and use of $\mathrm{BI}$ application intended for various analytical and reporting functionalities.

From the systemic aspect, DWH corresponds to an organizational scheme of a business system, from the data source level to the decision making level. From the technological aspect, DWH is a set of layers which provide reception, transformation, processing and on-line analytical data processing. DWH systems enable storage and processing of a large quantity of data from integrated heterogeneous sources in optimized multi-dimensional data schemes. DWH architecture of TMSS is shown in Fig. 3.

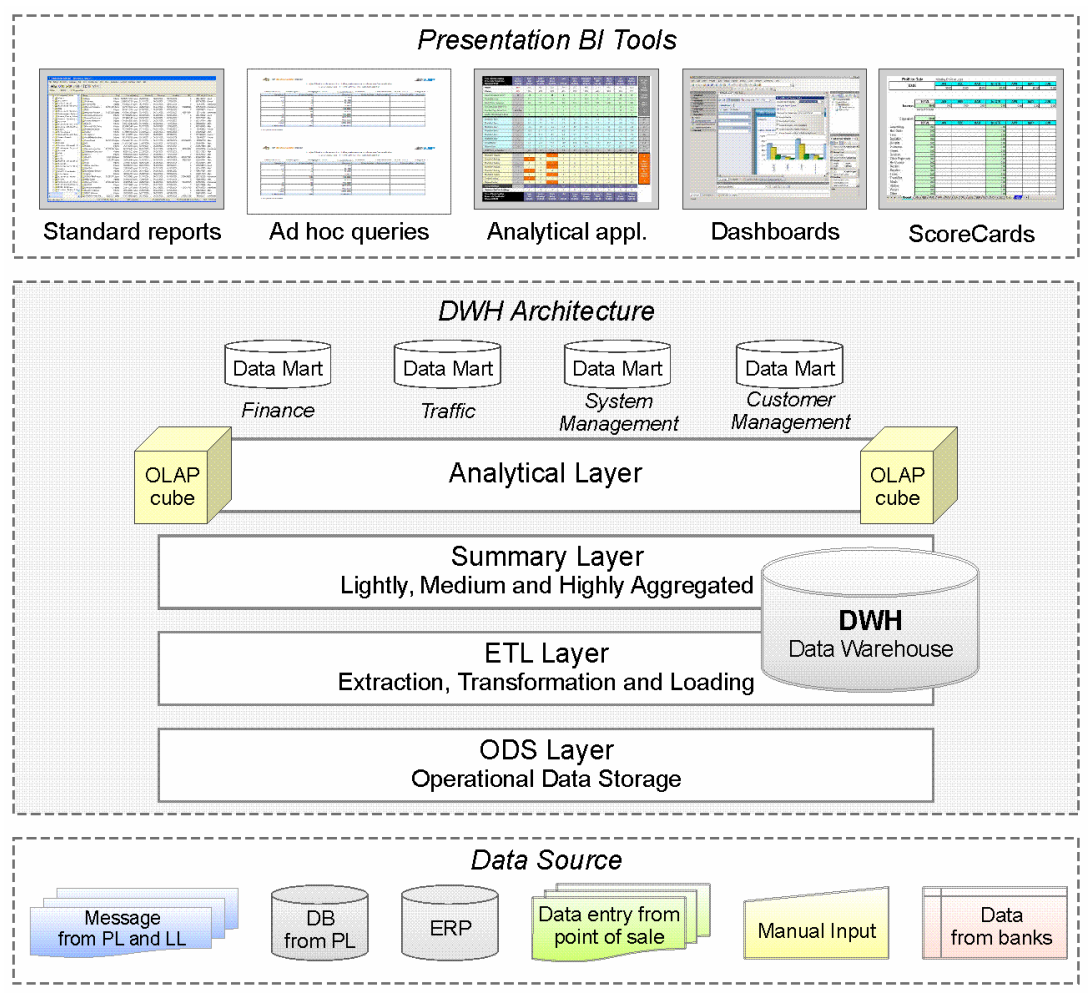

Fig. 3.

Data Warehouse Architecture

Data in DWH are collected from various internal and external sources: transaction messages from Toll plazas and Toll lanes, import data from Toll plaza data base for off-line regime, data obtained from ERP system, transaction messages and off-line entry of data from $\mathrm{OBU}$ and $\mathrm{CC}$ sales points, manual data input from documents, import of data from banks, and similar. The ODS (Operational Data Storage) layer receives and operationally stores all data from internal and external sources. The ETL layer 
(Extraction, Transformation and Loading) checks consistency of data in conformity with defined rules, establishes data integrity relations and fills data schemes in DWH. The Summary level aggregates data in various levels of detail (by hour, shift, day or any other period) in conformity with analytical data processing. The Analytical layer consists of a set of data models which present the basis for generating various reports, defined in the business system. The OLAP cube (Online Analytical Processing Cube) are data models designed in conformity with the requirements of online data processing. Data mart is a logical subset of DWH defined according to specific requirements of the business process or group of users, with an aim of obtaining various business reports which support making concrete decisions. In TMSS, Data Marts are designed according to basic system modules (Finance, Traffic,
System Management and Customer Management).

BI presentation tools enable development of BI applications which perform basic functionalities of the TMSS module Analytics and reporting - reporting on all segments of toll collection in conformity with the needs of business processes. Managers and TMSS users on different managerial levels monitor the toll collection system in on-line and off-line regime and control realization of all functions of the system through a set of reports. BI provides business information and analyzes key business processes, the making of good-quality decisions on different managerial levels and improvement of performances in the business system. The role of BI applications in the process of making business decisions is shown in Fig. 4.

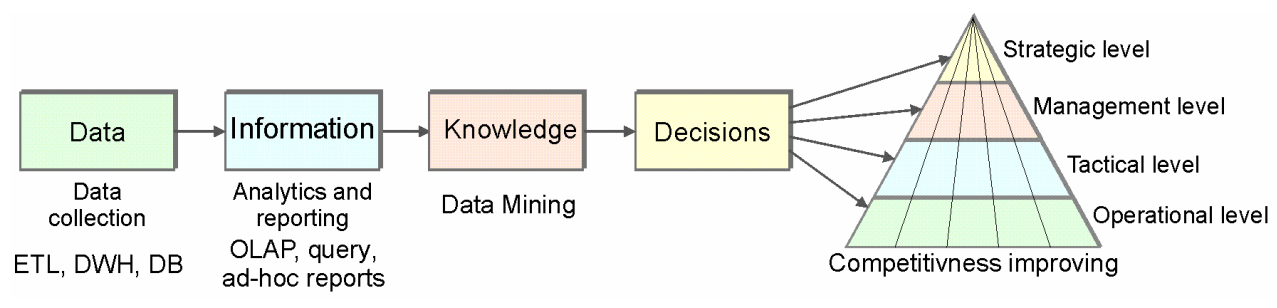

Fig. 4.

The Role of BI Systems in Decision Making

More than 40 reports have been developed using Oracle BI technology which have been grouped according to segments to which they refer (Radivojević et al., 2012): Toll collection (financial and traffic reports), OBU and CC, System Management and Customer Management. All reports can be obtained in various levels of detail, depending on the given input parameters (highway, toll plaza, toll collectors, period, medium, customer, and others).
BI platform enables development of different types of applications depending on data processing method, complexity, interaction with user and form of displaying information. The following types of BI applications have been developed in TMSS:

- $\quad$ Standard Reports - predefined reports, which use DWH system data through developed data models. They enable interaction with the users through 
input of set parameters which define reporting scope (highway, toll plaza, lane, period, toll collector, customer). This type of reporting is developed for functionalities necessary in everyday work of the system users (financial and traffic reports, customer reports, etc.).

- Ad-hoc queries and reports - enable data search for users through multidimensional data models (OLAP cube). Ad hoc reports give tabular result presentation; are made as needed and represent a response to the present requirements of the users.

- Analytical applications - complex reports which combine different sets of business information separated according to certain characteristics, with one or more approaches for information analysis. These applications refer to analytics of business processes and activities (reports on black and gray OBU and CC lists, traffic images of vehicle movements, reports on payments and vehicle passing with $\mathrm{OBU}$ and $\mathrm{CC}$, and others).

- Dashboards and Scorecards - represent the most advanced variant of BI applications because they include a large set of data which refer to different business processes and offer visualization of results in such a way that new information and knowledge is presented to the user in the fastest possible way. Dashboards are applied for supervision and management of processes and activities, providing monitoring of changes in real-time (overview of system state, overview of equipment state, and others). Balanced Scorecards enable monitoring of business performances from various aspects in real-time and warn on future changes (monitoring the work of Optical character recognition - OCR and video surveillance - VS system, communication of all system levels and monitoring data on inbound/outbound messages, and others).

All developed BI applications can have various formats of outbound data (doc, pdf, ppt, html, xml, xls), which can be displayed on screen, printed, e-mailed or faxed to users or stored in a repository. Applications can be carried out upon user's request, automatically in given time periods, or after certain actions in the business system. For example, report applications which are carried out every day for managing customers, bills and invoices for payment of OBU and $\mathrm{CC}$ which are sent to customers at the beginning of each month by e-mail, analytical applications which are carried out when OBU and CC black and gray lists are distributed to toll plazas, control panels which report on equipment operation in toll lanes, and others.

BI applications define the functioning of certain business activities and processes; they can have more or less detail and accordingly are intended for different managing levels of toll collection system. Based on such defined reports, high-quality decisions on individual business processes can be brought on each level, which refer to individual business processes, groups of processes or which refer to external systems (banks, OBU and CC providers, special customers, and others). Decisions made in the business system could refer to any of the following:

- Physical configuration of system (determining number and type of inbound and outbound lanes, defining work shifts),

- Tariff policy (toll pricelists, tariff 
packages for $\mathrm{OBU}$ and $\mathrm{CC}$, exemption lists and free passages),

- Stimulation for applying OBU and CC (increasing the network of sales points, bonuses and discounts for certain customers),

- Managing relations with customers (monitoring account state of customers and black and gray lists, introducing additional web services), and others.

\section{BI Effects in TMSS}

There are many advantages of applying BI technology, not only for the business system but for customers as well. According to certain research, BI enables a more precise insight into data and information, better comprehension of business changes, better planning and decision making on all levels (Russom, 2011).

The effects of applying BI in development and exploitation of TMSS-a can be viewed from many aspects:

- DWH is a contemporary data base technology necessary for application of BI, which enables storing of large quantities of data gathered from various sources. A well designed DWH system provides easy access to data on different levels of aggregation and data linking from different business processes.

- BI enables designing various applications, from standard predefined reports, to control panels used for monitoring performances and functioning of business processes in real-time. In the applications, it is possible to define different levels of interaction with users, the forms of visualizing information, the formats of outgoing results and time of execution.
- BI applications are carried out very quickly for large quantities of data and the most complex reports. Exploitation of TMSS so far has shown that the largest number of applications are almost immediately carried out while some complex analytical applications are carried out in less than $10 \mathrm{~s}$.

- Applications for supervision and management of equipment and toll collection processes in real-time enable linkage of various internal systems (OCR, VS, toll collection transactions) and good visualization of the process which improves supervision conditions to users and timely reacting.

- BI technology provides processing of all data in the toll collection system and their transformation into high-quality and reliable information which presents the basis for making decisions in all managing levels.

\section{Conclusion}

The application of BI in the Toll Management and Supervision System - TMSS is described in this paper. The effects of applying BI technology primarily include time savings in evaluating and analyzing performances of business processes, as well as a system of reporting which provides high-quality support in making decisions on all levels. Exploitation of TMSS to date has indicated to the positive effects of BI technology which could be perceived through the following:

- An improvement in the operational functioning of business processes - BI applications which provide monitoring, reporting and analytics of basic business processes; timely reactions in critical situations; making operative and tactical decisions. 
- An improvement in the managing process in the business system - strategic applications which provide reporting and analytics as support in making managerial and strategic decisions; measuring, monitoring, control and planning business performances.

BI technology is a new way of analyzing and methodology of designing DWH systems, and different types of BI applications, which correspond to the organizational structure of the business system from the data source level to the level of using new information and knowledge.

\section{Acknowledgements}

This work was supported by the Ministry of Education, Science and Technological Development of the Republic of Serbia through the project TR 36005, for the period 2011-2014.

\section{References}

Oracle. 2013. Oracle Event Processing. Available from Internet: <www.oracle.com/technetwork/middleware/ complex-event-processing/overview/ complex-eventprocessing-088095.html>.

Mihajlo Pupin Institute. 2012. Project Toll Charging System in Federation of Bosnia and Herzegovina - highway segment Jošanica-Kakanj. Mihajlo Pupin Institute, Belgrade, Serbia.

Radivojević, G.; Lazić, B.; Šormaz, G.; Kostić, P. 2013. Information System for Toll Management and Supervision. In Proceedings of the Symposium on operational research SYM-OP-IS 2013, Zlatibor, Serbia.
Radivojević, G.; Šormaz, G.; Kostić. P.; Lazić, B.; Šenborn, A. 2012. Data Analytics and Reporting in Toll Management and Supervision System - Case study Bosnia and Herzegovina. In Proceedings of the 2 nd International Conference on Supply Chain, 5 \& 6 October, Katerini, Greece.

Russom, P. 2011. Big Data Analytics, TDWI best practices Report. Available from Internet: <http://tdwi. org/research/list/tdwi-best-practices-reports.aspx $>$. 